\title{
Oscillation of third order nonlinear damped dynamic equation with mixed arguments on time scales
}

Ying Sui ${ }^{1}$ and Shurong Sun ${ }^{1 *}$

*Correspondence:

sshrong@163.com

'School of Mathematical Sciences,

University of Jinan, Jinan, P.R. China

\begin{abstract}
The objective of this paper is to offer sufficient conditions for the oscillation of all solutions of the third order nonlinear damped dynamic equation with mixed arguments of the form

$$
\left(r_{2}\left(r_{1}\left(y^{\Delta}\right)^{\alpha}\right)^{\Delta}\right)^{\Delta}(t)+p(t) \psi\left(t, y^{\Delta}(a(t))\right)+q(t) f(t, y(g(t)))=0
$$

on time scales, where $a(t) \geq t$ and $g(t) \leq t$. Using Riccati transformation, integral averaging technique, and comparison theorem, we give some new criteria for the oscillation of the studied equation. Our results essentially improve and complement the earlier ones.
\end{abstract}

MSC: 26E70; 34C10

Keywords: Time scales; Oscillation; Mixed arguments; Damped

\section{Introduction}

This paper deals with oscillatory behavior of all solutions of the third order nonlinear damped dynamic equation with mixed arguments of the form

$$
\left(r_{2}\left(r_{1}\left(y^{\Delta}\right)^{\alpha}\right)^{\Delta}\right)^{\Delta}(t)+p(t) \psi\left(t, y^{\Delta}(a(t))\right)+q(t) f(t, y(g(t)))=0, \quad t \in I,
$$

where $I=\left[t_{0}, \infty\right)_{\mathbb{T}}, \alpha \geq 1$ is the ratio of positive odd integers. In the sequel, assume that the conditions are satisfied:

(H1) $r_{1}, r_{2}, p, q \in C_{r d}\left(I, \mathbb{R}^{+}\right), a \in C_{r d}(I, \mathbb{R}), g \in C_{r d}^{1}(I, \mathbb{R})$, where $\mathbb{R}^{+}=(0, \infty)_{\mathbb{T}}$;

(H2) $a(t) \geq \sigma(t) \geq t, g(t) \leq t, g^{\Delta}(t) \geq 0$ and $g(t) \rightarrow \infty$ as $t \rightarrow \infty$;

(H3) $\psi, f \in C(\mathbb{T} \times \mathbb{R}, \mathbb{R})$ such that $\psi(t, x(t)) \geq k_{1} x^{\alpha}(t), \psi(t,-x(t))=-\psi(t, x(t))$, and $f(t, x(t)) \geq \max \left\{k_{2} x^{\beta}(t), k_{2} x^{\beta}(\sigma(t))\right\}, f(t,-x(t))=-f(t, x(t))$, and $x(t)$ is defined on $\mathbb{T}, k_{1}, k_{2}$ are constants, $\beta$ is the ratio of positive odd integers.

We define

$$
R_{1}\left(t, t_{1}\right)=\int_{t_{1}}^{t} \frac{\Delta s}{r_{1}^{1 / \alpha}(s)}, \quad R_{2}\left(t, t_{1}\right)=\int_{t_{1}}^{t} \frac{\Delta s}{r_{2}(s)}
$$

(c) The Author(s) 2018. This article is distributed under the terms of the Creative Commons Attribution 4.0 International License (http://creativecommons.org/licenses/by/4.0/), which permits unrestricted use, distribution, and reproduction in any medium, provided you give appropriate credit to the original author(s) and the source, provide a link to the Creative Commons license, and indicate if changes were made. 
and

$$
R^{*}\left(t, t_{1}\right)=\int_{t_{1}}^{t}\left(\frac{R_{2}\left(s, t_{1}\right)}{r_{1}(s)}\right)^{1 / \alpha} \Delta s
$$

for $t_{0} \leq t_{1} \leq t \leq \infty$, and assume that

$$
R_{1}\left(t, t_{0}\right) \rightarrow \infty, \quad t \rightarrow \infty
$$

and

$$
R_{2}\left(t, t_{0}\right) \rightarrow \infty, \quad t \rightarrow \infty
$$

Let $\mathbb{T}$ be a time scale with sup $\mathbb{T}=\infty$. We only consider these solutions of (1.1) which exist on some half-line $\left[t_{0}, \infty\right)_{\mathbb{T}}$ and satisfy $\sup \left\{|x(t)|: t_{1} \leq t<\infty\right\}>0$ for any $t_{1} \geq t_{0}$. If $y, r_{1}\left(y^{\Delta}\right)^{\alpha}, r_{2}\left(r_{1}\left(y^{\Delta}\right)^{\alpha}\right)^{\Delta} \in C_{r d}^{1}\left(\left[t_{y}, \infty\right), \mathbb{R}\right)$ and $y$ satisfies $(1.1)$ on $\left[t_{y}, \infty\right)_{\mathbb{T}}$ for some $t_{y} \geq t_{0}$, then the function $y$ is called a solution of (1.1). A solution $y(t)$ of (1.1) is said to be oscillatory if it is neither eventually positive nor eventually negative, otherwise it is called nonoscillatory. The equation itself is called oscillatory if all of its solutions are oscillatory.

In recent years, there has been an increasing interest in studying the oscillation of solutions of the equations, we refer the readers to [1-13] and the references cited therein. The dynamic equations with deviating arguments are deemed to be adequate in modeling of the countless processes in all areas of science. As is well known, a distinguishing feature of delay dynamic equations under consideration is the dependence of the evolution rate of the processes described by such equations on the past history. This consequently results in predicting the future in a more reliable and efficient way, explaining at the same time many qualitative phenomena such as periodicity, oscillation or instability. Contrariwise, advanced dynamic equations can find use in many applied problems whose evolution rate depends not only on the present, but also on the future, it also plays a vital role. The dynamic equations with mixed arguments have both advanced arguments and delay arguments, and have both properties.

In 2017, Baculíková [3] studied the oscillatory behavior of the second order advanced differential equation

$$
y^{\prime \prime}(t)+p(t) y(\sigma(t))=0
$$

where $\sigma(t) \geq t$, and amended some oscillatory criteria for the second order advanced differential equation.

And there are many results on the oscillation of the delay dynamic equation, we refer the readers to $[4-8,10-13]$ and the references cited therein. The study of dynamic equation with mixed arguments is also of great significance, due to the comprehensive use in natural science and theoretical study.

In 2014, Adivar et al. [8] studied the oscillation of the third order delay and advanced dynamic equations

$$
\left(\frac{1}{a(t)}\left(x^{\Delta}(t)\right)^{\alpha}\right)^{\Delta \Delta}+q(t) f(x[g(t)])=0
$$


and

$$
\left(\frac{1}{a(t)}\left(x^{\Delta}(t)\right)^{\alpha}\right)^{\Delta \Delta}=q(t) f(x[g(t)])+p(t) h(x[k(t)])
$$

on $\left[t_{0}, \infty\right)$ such that $t \in \mathbb{T}$ and $t_{0} \geq 0$, where $\alpha$ is the ratio of two positive odd integers.

However, to the best of our knowledge, there is very little known about the oscillatory behavior of dynamic equation with mixed arguments on time scales. And there are no known results regarding the oscillation of third order dynamic equation with mixed arguments of type (1.1). More exactly, the existing literature does not provide any criteria which ensure oscillation of all solutions of equation (1.1).

In view of the above motivation, our aim in this paper is to present sufficient conditions which ensure that all solutions of (1.1) are oscillatory. We give some new criteria for the oscillation of (1.1) by using the Riccati transformation and the integral averaging technique. Moreover, we present a new comparison theorem for deducing the oscillation of (1.1) from the oscillation of a suitable second order advanced dynamic equation. Thus, our method essentially simplifies the examination of the third order equation; and what is more, it supports backward the research on the second order advanced dynamic equation. For the study of oscillation of the advanced equation, we refer the readers to [3, 9, 10]. Indeed, there are no known results about the oscillation of the third order damped advanced dynamic equation in the form of $(1.1)$ when $q(t) \equiv 0$. And there are the results of the third order delay dynamic equation in the form of $(1.1)$ when $p(t) \equiv 0$. Our results essentially improve and complement the earlier ones. We also repair some of results of Bohner et al. [4].

\section{Preliminaries}

As usually, studying the properties of oscillatory solutions of (1.1), we can restrict our attention only to positive ones. In this section, we derive some new properties of oscillatory solutions of (1.1) that will be used for establishing new oscillatory criteria. Let

$$
\begin{array}{r}
L_{0} y(t)=y(t), \quad L_{1} y(t)=\left(r_{1}\left(y^{\Delta}\right)^{\alpha}\right)(t), \\
L_{2} y(t)=\left(r_{2}\left(r_{1}\left(y^{\Delta}\right)^{\alpha}\right)^{\Delta}\right)(t), \quad L_{3} y(t)=\left(r_{2}\left(r_{1}\left(y^{\Delta}\right)^{\alpha}\right)^{\Delta}\right)^{\Delta}(t) .
\end{array}
$$

Definition 2.1 For function $f: \mathbb{T} \rightarrow \mathbb{R}$, we define the derivative $f^{\Delta}$ as follows: Let $t \in \mathbb{T}$. If there exists a number $\alpha \in \mathbb{R}$ such that for all $\varepsilon>0$ there exists a neighborhood $U$ of $t$ with

$$
|f(\sigma(t))-f(s)-\alpha(\sigma(t)-s)| \leq \varepsilon|\sigma(t)-s|
$$

for all $s \in U$, then $f$ is said to be differentiable at $t$, and we call $\alpha$ the delta derivative of $f$ at $t$ and denote it by $f^{\Delta}(t)$.

Lemma 2.1 Assume that (1.1) is nonoscillatory and $y$ is a nonoscillatory solution of (1.1) on $\left[t_{1}, \infty\right)_{\mathbb{T}}, t_{1} \geq t_{0}$. Then there exists $t_{2} \in\left[t_{1}, \infty\right)_{\mathbb{T}}$ such that one of the following cases holds for all sufficiently large $t \geq t_{2}$ :
(1) $L_{3} y(t)<0$,
$L_{2} y(t)>0$,
$L_{1} y(t)>0$
(2) $L_{3} y(t)<0$,
$L_{2} y(t)>0$,
$L_{1} y(t)<0$. 
Proof If $y$ is a nonoscillatory solution of (1.1) on $\left[t_{1}, \infty\right)_{\mathbb{T}}$, say $y(t)>0, y(g(t))>0$ for $t \geq$ $t_{1} \geq t_{0}$. Since $p, q \in C_{r d}\left(I, \mathbb{R}^{+}\right), \psi, f \in C(\mathbb{T} \times \mathbb{R}, \mathbb{R})$, then, it is easy to see that

$$
L_{3} y(t)=-p(t) \psi\left(t, y^{\Delta}(a(t))\right)-q(t) f(t, y(g(t)))<0,
$$

then $L_{2} y(t)$ is decreasing on $\left[t_{1}, \infty\right)_{\mathbb{T}}$, which implies $L_{2} y(t)$ does not change sign eventually, then there exists $t_{2} \geq t_{1}$ such that either $L_{2} y(t)>0$ or $L_{2} y(t)<0$ for any $t \geq t_{2}$.

Next, assume that $L_{2} y(t)<0$, then $L_{1} y(t)$ is decreasing and $L_{1} y(t)>0$ or $L_{1} y(t)<0$ for $t \geq t_{3} \geq t_{2}$. If $L_{1} y(t)>0$, we have

$$
\begin{aligned}
L_{1} y(t) & =L_{1} y\left(t_{2}\right)+\int_{t_{2}}^{t}\left(L_{1} y\right)^{\Delta}(s) \Delta s=L_{1} y\left(t_{2}\right)+\int_{t_{2}}^{t} \frac{L_{2} y(s)}{r_{2}(s)} \Delta s \\
& \leq L_{1} y\left(t_{2}\right)+L_{2} y\left(t_{2}\right) \int_{t_{2}}^{t} \frac{1}{r_{2}(s)} \Delta s \\
& =L_{1} y\left(t_{2}\right)+L_{2} y\left(t_{2}\right) R_{2}\left(t, t_{2}\right) \quad \text { for any } t \geq t_{3} \geq t_{2},
\end{aligned}
$$

and (1.3) would imply $L_{1} y(t) \rightarrow-\infty$ as $t \rightarrow \infty$, which is a contradiction to the positivity of $L_{1} y(t)$. Further, if $L_{1} y(t)<0$, then by integration of

$$
y^{\Delta}(t)=\left(\frac{L_{1} y(t)}{r_{1}(t)}\right)^{1 / \alpha} \leq\left(\frac{L_{1} y\left(t_{2}\right)}{r_{1}(t)}\right)^{1 / \alpha}
$$

we obtain $y(t)<0$ for all large $t$, which is a contradiction. Altogether, $L_{2} y(t)>0$ on $\left[t_{3}, \infty\right)_{\mathbb{T}}$. This completes the proof.

Lemma 2.2 Suppose that (2.1) of Lemma 2.1 holds and $y$ is a nonoscillatory solution of (1.1), $t \geq t_{1} \geq t_{0}$. Then

$$
L_{1} y(t) \geq R_{2}\left(t, t_{1}\right) L_{2} y(t) \quad \text { for all } t \geq t_{1}
$$

and

$$
y(t) \geq R^{*}\left(t, t_{1}\right)\left(L_{2} y\right)^{1 / \alpha}(t) \quad \text { for all } t \geq t_{1} .
$$

Proof If $y$ is a nonoscillatory solution of (1.1), and $y(t)>0, y(g(t))>0$ for $t \geq t_{1} \geq t_{0}$. Since $L_{1} y(t)>0$, then

$$
\begin{aligned}
L_{1} y(t) & =L_{1} y\left(t_{1}\right)+\int_{t_{1}}^{t}\left(L_{1} y\right)^{\Delta}(s) \Delta s \geq \int_{t_{1}}^{t}\left(L_{1} y\right)^{\Delta}(s) \Delta s \\
& =\int_{t_{1}}^{t} \frac{L_{2} y(s)}{r_{2}(s)} \Delta s \geq \int_{t_{1}}^{t} \frac{L_{2} y(t)}{r_{2}(s)} \Delta s=R_{2}\left(t, t_{1}\right) L_{2} y(t) .
\end{aligned}
$$

Thus

$$
y^{\Delta}(t) \geq\left(\frac{R_{2}\left(t, t_{1}\right)}{r_{1}(t)}\right)^{1 / \alpha}\left(L_{2} y\right)^{1 / \alpha}(t) .
$$


Now, integrating the above inequality from $t_{1}$ to $t$, we have

$$
\begin{aligned}
y(t) & =y\left(t_{1}\right)+\int_{t_{1}}^{t} y^{\Delta}(s) \Delta s \geq \int_{t_{1}}^{t} y^{\Delta}(s) \Delta s \geq \int_{t_{1}}^{t}\left(\frac{R_{2}\left(s, t_{1}\right)}{r_{1}(s)}\right)^{1 / \alpha}\left(L_{2} y\right)^{1 / \alpha}(s) \Delta s \\
& \geq\left[\int_{t_{1}}^{t}\left(\frac{R_{2}\left(s, t_{1}\right)}{r_{1}(s)}\right)^{1 / \alpha} \Delta s\right]\left(L_{2} y\right)^{1 / \alpha}(t)=R^{*}\left(t, t_{1}\right)\left(L_{2} y\right)^{1 / \alpha}(t) \quad \text { for } t \geq t_{1} .
\end{aligned}
$$

This completes the proof.

Lemma $2.3([11,12])$ Assume that $\beta>0$ is the ratio of positive odd integers and $x^{\beta}(t) \in$ $C_{r d}^{1}(I, \mathbb{R})$. Then

$$
\left(x^{\beta}(t)\right)^{\Delta} \geq \begin{cases}\beta(x(\sigma(t)))^{\beta-1} x^{\Delta}(t), & 0<\beta \leq 1 \\ \beta(x(t))^{\beta-1} x^{\Delta}(t), & \beta \geq 1\end{cases}
$$

Lemma 2.4 ([14] (Theorem 1.14) (Mean value theorem)) Let $f$ be a continuous function on $[a, b]$ that is differentiable on $[a, b)$. Then there exist $\eta, \xi \in[a, b)$ such that

$$
f^{\Delta}(\xi) \leq \frac{f(b)-f(a)}{b-a} \leq f^{\Delta}(\eta)
$$

\section{Oscillation results}

Now we are prepared to provide our main oscillatory theorems. By using the Riccati transformation and the integral averaging technique due to Philos [15], we establish new oscillation results for (1.1). Firstly, let us introduce now the class of functions $\mathcal{P}$ which will be used in this section. Let

$$
D_{0}=\left\{(t, s)_{\mathbb{T}}: t>s>t_{0}\right\} \quad \text { and } \quad D=\left\{(t, s)_{\mathbb{T}}: t \geq s>t_{0}\right\}
$$

A function $H \in C_{r d}(D, \mathbb{R})$ is said to belong to the class $\mathcal{P}$ if

$$
\begin{cases}H(t, s)>0, & (t, s)_{\mathbb{T}} \in D_{0} \\ H(t, s)=0, & s=t\end{cases}
$$

and $H(t, s)$ has a continuous and nonpositive partial derivative on $D_{0}$ with respect to the second variable, and for a positive continuous function $\bar{h}$,

$$
-H^{\Delta_{s}}(t, s)=\bar{h}(t, s) \sqrt{H(t, s)}, \quad(t, s)_{\mathbb{T}} \in D_{0}
$$

When $H(t, s)=(t-s)^{n}, n \in N$, the Philos-type conditions reduce to the Kamenev-type ones.

Theorem 3.1 Assume that (1.2) (1.3) hold, $\alpha \geq \beta$. If there exist a function $m \in C_{r d}(I, \mathbb{R})$ such that $m(t)>0$ and a function $H(t, s) \in \mathcal{P}$ satisfying

$$
\limsup _{t \rightarrow \infty} \frac{1}{H\left(t, t_{1}\right)} \int_{t_{1}}^{t}\left[k_{2} m(s) q(s) H(t, s)-\frac{P^{2}(t, s)}{4 B(s)}\right] \Delta s=\infty \quad \text { for all large } t \geq t_{1}
$$


where

$$
P(t, s)=\bar{h}(t, s)-A(s) \sqrt{H(t, s)},
$$

and

$$
\left\{\begin{array}{l}
A(t)=\frac{m^{\Delta}(t)}{m(\sigma(t))}-\frac{k_{1} m(t) p(t)}{m(\sigma(t)) r_{1}(a(t))} R_{2}\left(\sigma(t), t_{1}\right), \\
B(t)=c^{*} m(t) m^{-2}(\sigma(t)) g^{\Delta}(t)\left(R^{*}\left(g(\sigma(t)), t_{1}\right)\right)^{\beta-1}\left(\frac{R_{2}\left(g(t), t_{1}\right)}{r_{1}(\xi)}\right)^{1 / \alpha}
\end{array}\right.
$$

Moreover, if every solution of the equation

$$
\left(r_{2} z^{\Delta}\right)^{\Delta}(t)-Q(t) z(a(t))=0
$$

is oscillatory, where

$$
Q(t)=c k_{2} q(t)\left(R_{1}(a(t), g(t))\right)^{\beta}-\frac{k_{1} p(t)}{r_{1}(a(t))}, \quad t \geq t_{1},
$$

for all constants $c, c^{*}>0$. Then every solution $y(t)$ of $(1.1)$ or $L_{2} y(t)$ is oscillatory.

Proof If $y$ is a nonoscillatory solution of $(1.1)$ on $\left[t_{1}, \infty\right)_{\mathbb{T}}, t_{1} \geq t_{0}$. Assume that $y(t)>0$ and $y(g(t))>0$ for $t \geq t_{1}$. By the proof of Lemma 2.1, we have that two cases of Lemma 2.1 hold. Now, we shall show that in each case we are led to a contradiction.

Case (1). Suppose that (2.1) of Lemma 2.1 holds. Define the following Riccati transformation:

$$
w(t)=m(t) \frac{L_{2} y(t)}{y^{\beta}(g(t))}, \quad t \in\left[t_{1}, \infty\right)_{\mathbb{T}} .
$$

Then $w(t)>0$, and

$$
\begin{aligned}
w^{\Delta}(t) & =\left[m(t) \frac{L_{2} y(t)}{y^{\beta}(g(t))}\right]^{\Delta} \\
& =m^{\Delta}(t) \frac{L_{2} y(\sigma(t))}{y^{\beta}(g(\sigma(t)))}+m(t) \frac{\left(L_{2} y\right)^{\Delta}(t) y^{\beta}(g(t))-L_{2} y(t)\left[y^{\beta}(g(t))\right]^{\Delta}}{y^{\beta}(g(t)) y^{\beta}(g(\sigma(t)))} \\
& =\frac{m^{\Delta}(t)}{m(\sigma(t))} w(\sigma(t))+m(t) \frac{\left(L_{2} y\right)^{\Delta}(t)}{y^{\beta}(g(\sigma(t)))}-m(t) \frac{L_{2} y(t)\left[y^{\beta}(g(t))\right]^{\Delta}}{y^{\beta}(g(t)) y^{\beta}(g(\sigma(t)))} \\
& =\frac{m^{\Delta}(t)}{m(\sigma(t))} w(\sigma(t))+\frac{m(t)}{m(\sigma(t))} \frac{\left(L_{2} y\right)^{\Delta}(t)}{L_{2} y(\sigma(t))} w(\sigma(t))-m(t) \frac{L_{2} y(t)\left[y^{\beta}(g(t))\right]^{\Delta}}{y^{\beta}(g(t)) y^{\beta}(g(\sigma(t)))} .
\end{aligned}
$$

By (H3) and $y(g(\sigma(t))) \geq y(g(t))$, we have $f(t, y(g(t))) \geq k_{2} y^{\beta}(g(\sigma(t)))$. From (1.1) and (2.4), then

$$
\begin{aligned}
& \frac{m^{\Delta}(t)}{m(\sigma(t))} w(\sigma(t))+\frac{m(t)}{m(\sigma(t))} \frac{\left(L_{2} y\right)^{\Delta}(t)}{L_{2} y(\sigma(t))} w(\sigma(t)) \\
& \quad \leq \frac{m^{\Delta}(t)}{m(\sigma(t))} w(\sigma(t))-\frac{m(t)}{m(\sigma(t))} \frac{\frac{k_{1} p(t)}{r_{1}(a(t))} L_{1} y(a(t))+q(t) f(t, y(g(t)))}{L_{2} y(\sigma(t))} w(\sigma(t))
\end{aligned}
$$




$$
\begin{aligned}
& \leq \frac{m^{\Delta}(t)}{m(\sigma(t))} w(\sigma(t))-\frac{\frac{k_{1} p(t)}{r_{1}(a(t))} L_{1} y(\sigma(t)) m(t)}{m(\sigma(t)) L_{2} y(\sigma(t))} w(\sigma(t))-\frac{m(t) q(t) f(t, y(g(t)))}{m(\sigma(t)) L_{2} y(\sigma(t))} w(\sigma(t)) \\
& \leq \frac{m^{\Delta}(t)}{m(\sigma(t))} w(\sigma(t))-\frac{k_{1} m(t) p(t)}{m(\sigma(t)) r_{1}(a(t))} R_{2}\left(\sigma(t), t_{1}\right) w(\sigma(t))-k_{2} m(t) q(t) \\
& =\left[\frac{m^{\Delta}(t)}{m(\sigma(t))}-\frac{k_{1} m(t) p(t)}{m(\sigma(t)) r_{1}(a(t))} R_{2}\left(\sigma(t), t_{1}\right)\right] w(\sigma(t))-k_{2} m(t) q(t) \\
& =A(t) w(\sigma(t))-k_{2} m(t) q(t) .
\end{aligned}
$$

Now, according to the method given in [16], and by Lemma 2.3, we have

$$
\left(y^{\beta}(g(t))\right)^{\Delta} \geq \begin{cases}\beta(y(g(\sigma(t))))^{\beta-1}(y(g(t)))^{\Delta}, & 0<\beta \leq 1, \\ \beta(y(g(t)))^{\beta-1}(y(g(t)))^{\Delta}, & \beta \geq 1 .\end{cases}
$$

Then, if $\sigma(t)>t$, by Lemma 2.4, we get

$$
(y(g(t)))^{\Delta}=\frac{y(g(\sigma(t)))-y(g(t))}{\sigma(t)-t}=\frac{y(g(\sigma(t)))-y(g(t))}{g(\sigma(t))-g(t)} g^{\Delta}(t) \geq y^{\Delta}(\xi) g^{\Delta}(t),
$$

where $\xi \in[g(t), g(\sigma(t)))$. If $\sigma(t)=t$, we obtain $g(\sigma(t))=\sigma(g(t))=g(t)$ and

$$
\left(y^{\beta}(g(t))\right)^{\Delta}=y^{\prime}(g(t)) g^{\prime}(t) .
$$

Moreover, since $L_{2} y(t)>0$, which implies that $r_{1}(t)\left(y^{\Delta}(t)\right)^{\alpha}$ is increasing, then

$$
r_{1}(\xi)\left(y^{\Delta}(\xi)\right)^{\alpha} \geq r_{1}(g(t))\left(y^{\Delta}(g(t))\right)^{\alpha}
$$

that is,

$$
y^{\Delta}(\xi) \geq\left(\frac{r_{1}(g(t))}{r_{1}(\xi)}\right)^{1 / \alpha} y^{\Delta}(g(t))
$$

thus

$$
(y(g(t)))^{\Delta} \geq\left(\frac{r_{1}(g(t))}{r_{1}(\xi)}\right)^{1 / \alpha} y^{\Delta}(g(t)) g^{\Delta}(t) .
$$

Then, for $0<\beta \leq 1$,

$$
\begin{aligned}
-m(t) \frac{L_{2} y(t)\left[y^{\beta}(g(t))\right]^{\Delta}}{y^{\beta}(g(t)) y^{\beta}(g(\sigma(t)))} & \leq-m(\sigma(t)) \frac{m(t) L_{2} y(\sigma(t))\left[y^{\beta}(g(t))\right]^{\Delta}}{m(\sigma(t)) y^{2 \beta}(g(\sigma(t)))} \\
& =-w(\sigma(t)) \frac{m(t)\left[y^{\beta}(g(t))\right]^{\Delta}}{m(\sigma(t)) y^{\beta}(g(\sigma(t)))} \\
& \leq-w(\sigma(t)) \frac{m(t) \beta y^{\beta-1}(g(\sigma(t))) g^{\Delta}(t) r_{1}^{1 / \alpha}(g(t)) y^{\Delta}(g(t))}{m(\sigma(t)) y^{\beta}(g(\sigma(t))) r_{1}^{1 / \alpha}(\xi)} \\
& =-\beta w(\sigma(t)) \frac{m(t) g^{\Delta}(t) r_{1}^{1 / \alpha}(g(t)) y^{\Delta}(g(t))}{m(\sigma(t)) y(g(\sigma(t))) r_{1}^{1 / \alpha}(\xi)}, \quad 0<\beta \leq 1 .
\end{aligned}
$$


And for $\beta \geq 1$,

$$
\begin{aligned}
-m(t) \frac{L_{2} y(t)\left[\left(y^{\beta}(g(t))\right]^{\Delta}\right.}{y^{\beta}(g(t)) y^{\beta}(g(\sigma(t)))} & \leq-m(\sigma(t)) \frac{m(t) L_{2} y(\sigma(t))\left[y^{\beta}(g(t))\right]^{\Delta}}{m(\sigma(t)) y^{\beta}(g(t)) y^{\beta}(g(\sigma(t)))} \\
& =-w(\sigma(t)) \frac{m(t)\left[y^{\beta}(g(t))\right]^{\Delta}}{m(\sigma(t)) y^{\beta}(g(t))} \\
& \leq-w(\sigma(t)) \frac{m(t) \beta y^{\beta-1}(g(t)) g^{\Delta}(t) r_{1}^{1 / \alpha}(g(t)) y^{\Delta}(g(t))}{m(\sigma(t)) y^{\beta}(g(t)) r_{1}^{1 / \alpha}(\xi)} \\
& =-w(\sigma(t)) \frac{m(t) \beta g^{\Delta}(t) r_{1}^{1 / \alpha}(g(t)) y^{\Delta}(g(t))}{m(\sigma(t)) y(g(t)) r_{1}^{1 / \alpha}(\xi)} \\
& \leq-\beta w(\sigma(t)) \frac{m(t) g^{\Delta}(t) r_{1}^{1 / \alpha}(g(t)) y^{\Delta}(g(t))}{m(\sigma(t)) y(g(\sigma(t))) r_{1}^{1 / \alpha}(\xi)}, \quad \beta \geq 1 .
\end{aligned}
$$

Altogether, for all $\beta>0$, one has

$$
-m(t) \frac{L_{2} y(t)\left[y^{\beta}(g(t))\right]^{\Delta}}{y^{\beta}(g(t)) y^{\beta}(g(\sigma(t)))} \leq-\beta w(\sigma(t)) \frac{m(t) y^{\Delta}(g(t)) g^{\Delta}(t) r_{1}^{1 / \alpha}(g(t))}{m(\sigma(t)) y(g(\sigma(t))) r_{1}^{1 / \alpha}(\xi)} .
$$

Then (3.4) implies that

$$
w^{\Delta}(t) \leq A(t) w(\sigma(t))-k_{2} m(t) q(t)-\beta w(\sigma(t)) \frac{m(t) y^{\Delta}(g(t)) g^{\Delta}(t) r_{1}^{1 / \alpha}(g(t))}{m(\sigma(t)) y(g(\sigma(t))) r_{1}^{1 / \alpha}(\xi)} .
$$

By (2.4), we have

$$
\begin{aligned}
y^{\Delta}(g(t)) & =\left(\frac{1}{r_{1}(g(t))} L_{1} y(g(t))\right)^{1 / \alpha} \geq\left(\frac{R_{2}\left(g(t), t_{1}\right)}{r_{1}(g(t))}\right)^{1 / \alpha}\left(L_{2} y(g(t))\right)^{1 / \alpha} \\
& \geq\left(\frac{R_{2}\left(g(t), t_{1}\right)}{r_{1}(g(t))}\right)^{1 / \alpha}\left(L_{2} y(\sigma(t))\right)^{1 / \alpha} .
\end{aligned}
$$

Further,

$$
\begin{aligned}
\frac{y^{\Delta}(g(t))}{y(g(\sigma(t)))} \geq & \left(\frac{R_{2}\left(g(t), t_{1}\right)}{m(\sigma(t)) r_{1}(g(t))}\right)^{1 / \alpha} \frac{m^{1 / \alpha}(\sigma(t))\left(L_{2} y\right)^{1 / \alpha}(\sigma(t))}{y^{\beta / \alpha}(g(\sigma(t)))} y^{\beta / \alpha-1}(g(\sigma(t))) \\
& \stackrel{(3.4)}{=}\left(\frac{R_{2}\left(g(t), t_{1}\right)}{m(\sigma(t)) r_{1}(g(t))}\right)^{1 / \alpha} w^{1 / \alpha}(\sigma(t)) y^{\beta / \alpha-1}(g(\sigma(t))) .
\end{aligned}
$$

Then (3.6) implies that

$$
\begin{aligned}
w^{\Delta}(t) \leq & A(t) w(\sigma(t))-k_{2} m(t) q(t) \\
& -\beta w^{1 / \alpha+1}(\sigma(t)) y^{\beta / \alpha-1}(g(\sigma(t))) \frac{m(t) g^{\Delta}(t) R_{2}^{1 / \alpha}\left(g(t), t_{1}\right)}{m^{1 / \alpha+1}(\sigma(t)) r_{1}^{1 / \alpha}(\xi)} .
\end{aligned}
$$


What is more,

$$
\begin{aligned}
r_{1}(t)\left(y^{\Delta}\right)^{\alpha}(t) & =L_{1} y(t)=L_{1} y\left(t_{1}\right)+\int_{t_{1}}^{t}\left(L_{1} y\right)^{\Delta}(s) \Delta s \leq L_{1} y\left(t_{1}\right)+c_{1} \int_{t_{1}}^{t} \frac{\Delta s}{r_{2}(s)} \\
& =L_{1} y\left(t_{1}\right)+c_{1} R_{2}\left(t, t_{1}\right)=\left[\frac{L_{1} y\left(t_{1}\right)}{R_{2}\left(t, t_{1}\right)}+c_{1}\right] R_{2}\left(t, t_{1}\right) \\
& \leq\left[\frac{L_{1} y\left(t_{1}\right)}{R_{2}\left(t_{2}, t_{1}\right)}+c_{1}\right] R_{2}\left(t, t_{1}\right)=\tilde{c}_{1} R_{2}\left(t, t_{1}\right)
\end{aligned}
$$

holds for all $t \geq t_{2}$, where $c_{1}=L_{2} y\left(t_{1}\right)$ and $\tilde{c}_{1}=c_{1}+\frac{L_{1} y\left(t_{1}\right)}{R_{2}\left(t_{2}, t_{1}\right)}$. And

$$
\begin{aligned}
y(t) & =y\left(t_{2}\right)+\int_{t_{2}}^{t} y^{\Delta}(s) \Delta s \leq y\left(t_{2}\right)+\int_{t_{2}}^{t}\left(\frac{\tilde{c}_{1} R_{2}\left(s, t_{1}\right)}{r_{1}(s)}\right)^{1 / \alpha} \Delta s \\
& \leq y\left(t_{2}\right)+\int_{t_{1}}^{t}\left(\frac{\tilde{c}_{1} R_{2}\left(s, t_{1}\right)}{r_{1}(s)}\right)^{1 / \alpha} \Delta s=y\left(t_{2}\right)+\tilde{c}_{1}^{1 / \alpha} R^{*}\left(t, t_{1}\right) \\
& =\left[\frac{y\left(t_{2}\right)}{R^{*}\left(t, t_{1}\right)}+\tilde{c}_{1}^{1 / \alpha}\right] R^{*}\left(t, t_{1}\right) \leq\left[\frac{y\left(t_{2}\right)}{R^{*}\left(t_{2}, t_{1}\right)}+\tilde{c}_{1}^{1 / \alpha}\right] R^{*}\left(t, t_{1}\right)=c_{2} R^{*}\left(t, t_{1}\right)
\end{aligned}
$$

holds for all $t \geq t_{2} \geq t_{1}$, where $c_{2}=\frac{y\left(t_{2}\right)}{R^{*}\left(t_{2}, t_{1}\right)}+\tilde{c}_{1}^{1 / \alpha}$. By (3.3) and (2.5), we have

$$
w(t)=m(t) \frac{L_{2} y(t)}{y^{\beta}(g(t))} \leq m(t) \frac{L_{2} y(g(t))}{y^{\beta}(g(t))} \leq m(t)\left(R^{*}\left(g(t), t_{1}\right)\right)^{-\alpha} y^{\alpha-\beta}(g(t)), \quad t \geq t_{1} .
$$

Using (3.8) in (3.9), we get

$$
w(t) \leq c_{2}^{\alpha-\beta} m(t)\left(R^{*}\left(g(t), t_{1}\right)\right)^{-\beta}, \quad t \geq t_{2} .
$$

Using (3.8) and (3.10) in (3.7), we obtain

$$
\begin{aligned}
w^{\Delta}(t) \leq & A(t) w(\sigma(t))-k_{2} m(t) q(t) \\
& -w^{2}(\sigma(t))\left[\beta c_{2}^{\beta-\alpha} m(t) m^{-2}(\sigma(t)) g^{\Delta}(t)\left(R^{*}\left(g(\sigma(t)), t_{1}\right)\right)^{\beta-1}\left(\frac{R_{2}\left(g(t), t_{1}\right)}{r_{1}(\xi)}\right)^{1 / \alpha}\right] \\
\leq & A(t) w(\sigma(t))-k_{2} m(t) q(t)-B(t) w^{2}(\sigma(t)), \quad t \geq t_{2},
\end{aligned}
$$

where $c^{*}=\beta c_{2}^{\beta-\alpha}$.

Next,

$$
\begin{aligned}
& \int_{t_{1}}^{t} k_{2} m(s) q(s) H(t, s) \Delta s \\
& \quad \leq \int_{t_{1}}^{t} H(t, s)\left\{-w^{\Delta}(s)+A(s) w(\sigma(s))-B(s) w^{2}(\sigma(s))\right\} \Delta s \\
& \quad=-\left.H(t, s) w(s)\right|_{s=t_{1}} ^{s=t}+\int_{t_{1}}^{t}\left\{H^{\Delta_{s}}(t, s) w(\sigma(s))+H(t, s)\left[A(s) w(\sigma(s))-B(s) w^{2}(\sigma(s))\right]\right\} \Delta s
\end{aligned}
$$




$$
\begin{aligned}
& =H\left(t, t_{1}\right) w\left(t_{1}\right)-\int_{t_{1}}^{t}\left\{H(t, s) B(s) w^{2}(\sigma(s))+w(\sigma(s))[\bar{h}(t, s) \sqrt{H(t, s)}-H(t, s) A(s)]\right\} \Delta s \\
& =H\left(t, t_{1}\right) w\left(t_{1}\right)-\int_{t_{1}}^{t}\left\{\sqrt{H(t, s)} \sqrt{B(s)} w(\sigma(s))+\frac{P(t, s)}{2 \sqrt{B(s)}}\right\}^{2} \Delta s+\int_{t_{1}}^{t} \frac{P^{2}(t, s)}{4 B(s)} \Delta s \\
& \leq H\left(t, t_{1}\right) w\left(t_{1}\right)+\int_{t_{1}}^{t} \frac{P^{2}(t, s)}{4 B(s)} \Delta s .
\end{aligned}
$$

Therefore,

$$
\frac{1}{H\left(t, t_{1}\right)} \int_{t_{1}}^{t}\left[k_{2} m(s) q(s) H(t, s)-\frac{P^{2}(t, s)}{4 B(s)}\right] \Delta s \leq w\left(t_{1}\right)
$$

which contradicts with (3.1).

Case (2). Suppose that (2.2) of Lemma 2.1 holds. Now, for $v \geq u \geq t_{2}$, we have

$$
\begin{aligned}
y(u) & >y(u)-y(v) \\
& =-\int_{u}^{v} r_{1}^{-1 / \alpha}(\tau)\left(r_{1}(\tau)\left(y^{\Delta}(\tau)\right)^{\alpha}\right)^{1 / \alpha} \Delta \tau \geq\left(\int_{u}^{v} r_{1}^{-1 / \alpha}(\tau) \Delta \tau\right)\left(-L_{1} y(v)\right)^{1 / \alpha} \\
& =R_{1}(v, u)\left(-L_{1} y(v)\right)^{1 / \alpha} .
\end{aligned}
$$

Letting $u=g(t)$ and $v=a(t)$,

$$
y(g(t))>R_{1}(a(t), g(t))\left(-L_{1} y(a(t))\right)^{1 / \alpha}=R_{1}(a(t), g(t)) x(a(t))
$$

for $a(t) \geq g(t) \geq t_{2}$, where $x(t)=\left(-L_{1} y(t)\right)^{1 / \alpha}>0$ for $t \geq t_{2}$. By (H3) and $y(g(t)) \geq y(g(\sigma(t)))$, we have $f(t, y(g(t))) \geq k_{2} y^{\beta}(g(t))$. Then from (1.1) and combined with the fact that $x(t)$ is decreasing, we get

$$
\left(r_{2} z^{\Delta}\right)^{\Delta}(t)+\frac{k_{1} p(t)}{r_{1}(a(t))} z(a(t)) \geq k_{2} q(t)\left(R_{1}(a(t), g(t))\right)^{\beta} z(a(t)) z^{\beta / \alpha-1}(a(t)),
$$

where $z(t)=x^{\alpha}(t)>0$. Since $z(t)$ is decreasing and $\alpha \geq \beta$, there exists a constant $c_{4}>0$ such that $z^{\beta / \alpha-1}(t) \geq c_{4}$ for $t \geq t_{2}$. Then

$$
\begin{aligned}
\left(r_{2} z^{\Delta}\right)^{\Delta}(t) & \geq k_{2} q(t)\left(R_{1}(a(t), g(t))\right)^{\beta} z(a(t)) z^{\beta / \alpha-1}(a(t))-\frac{k_{1} p(t)}{r_{1}(a(t))} z(a(t)) \\
& \geq\left[c_{4} k_{2} q(t)\left(R_{1}(a(t), g(t))\right)^{\beta}-\frac{k_{1} p(t)}{r_{1}(a(t))}\right] z(a(t)) .
\end{aligned}
$$

This gives

$$
\left(r_{2} z^{\Delta}\right)^{\Delta}(t) \geq Q(t) z(a(t))
$$

then

$$
\left(r_{2} z^{\Delta}\right)^{\Delta}(t)-Q(t) z(a(t)) \geq 0 .
$$


And $z(t)$ is an eventually positive solution of inequation (3.14). Integrating $y(t)=-z^{\Delta}(t)>0$ from $t_{1}$ to $t \geq t_{1}$, we obtain

$$
z(t)=z\left(t_{1}\right)-\int_{t_{1}}^{t} y(s) \Delta s
$$

then we have

$$
z(a(t))=z\left(t_{1}\right)-\int_{t_{1}}^{a(t)} y(s) \Delta s
$$

and (3.14) can be written as

$$
\left(r_{2} y\right)^{\Delta}(t)+Q(t)\left(z\left(t_{1}\right)-\int_{t_{1}}^{a(t)} y(s) \Delta s\right) \leq 0, \quad t \geq t_{1} .
$$

Integrating (3.15) from $t$ to $u \geq t \geq t_{1}$ and $u \rightarrow \infty$, we obtain

$$
y(t) \geq \frac{1}{r_{2}(t)} \int_{t}^{+\infty} Q(s)\left(z\left(t_{1}\right)-\int_{t_{1}}^{a(s)} y(\tau) \Delta \tau\right) \Delta s
$$

Now define the sequence $\left\{x_{j}(t)\right\}_{j \in N_{0}}: x_{0}(t)=y(t)$ :

$$
x_{j+1}(t)=\frac{1}{r_{2}(t)} \int_{t}^{+\infty} Q(s)\left(z\left(t_{1}\right)-\int_{t_{1}}^{a(s)} x_{j}(\tau) \Delta \tau\right) \Delta s, \quad j \in N_{0}, t \geq t_{1} .
$$

Then by (3.16) we get

$$
0<x_{j}(t) \leq y(t), \quad \text { and } \quad x_{j+1}(t) \leq x_{j}(t), \quad j \in N_{0}, t \geq t_{1} .
$$

So we obtain that the sequence $\left\{x_{j}(t)\right\}_{j \in N_{0}}$ is positive and nonincreasing on $j$. Then we define

$$
x(t)=\lim _{j \rightarrow \infty} x_{j}(t) \geq 0 .
$$

By the Lebesgue control convergence theorem [17], from (3.17), we have

$$
r_{2}(t) x(t)=\int_{t}^{+\infty} Q(s)\left(z\left(t_{1}\right)-\int_{t_{1}}^{a(s)} x(\tau) \Delta \tau\right) \Delta s
$$

then

$$
\left(r_{2}(t) x(t)\right)^{\Delta}=-Q(t)\left(z\left(t_{1}\right)-\int_{t_{1}}^{a(t)} x(s) \Delta s\right) .
$$

Let

$$
v(t)=z\left(t_{1}\right)-\int_{t_{1}}^{t} x(s) \Delta s>0
$$


and

$$
v^{\Delta}(t)=-x(t)
$$

From (3.18) we get

$$
\left(r_{2}(t) v^{\Delta}(t)\right)^{\Delta}=-\left(r_{2}(t) x(t)\right)^{\Delta}=Q(t) v(a(t))
$$

where

$$
v(a(t))=z\left(t_{1}\right)-\int_{t_{1}}^{a(t)} x(s) \Delta s .
$$

By (3.19), (3.20), we get

$$
\left(r_{2} v^{\Delta}\right)^{\Delta}(t)-Q(t) v(a(t))=0
$$

So $v$ is a positive solution of (3.2), which contradicts with (3.2) is oscillatory. This completes the proof.

Theorem 3.2 Assume that the hypotheses of Theorem 3.1 hold, except (3.1). Moreover, suppose that, for all $t \in I$,

$$
\limsup _{t \rightarrow \infty} \int_{t_{1}}^{t}\left[k_{2} m(s) q(s)-\frac{A^{2}(s)}{4 B(s)}\right] \Delta s=\infty
$$

Then every solution $y(t)$ of $(1.1)$ or $L_{2} y(t)$ is oscillatory.

Proof If $y$ is a nonoscillatory solution of $(1.1)$ on $\left[t_{1}, \infty\right)_{\mathbb{T}}$. Assume that $y(t)>0$ and $y(g(t))>$ 0 for $t \geq t_{1}$. By the proof of Lemma 2.1, we have that two cases of Lemma 2.1 hold.

Case (1). Suppose that (2.1) of Lemma 2.1 holds, then proceeding as in the proof of Theorem 3.1, we obtain (3.11), then

$$
\begin{aligned}
w^{\Delta}(t) & \leq A(t) w(\sigma(t))-k_{2} m(t) q(t)-B(t) w^{2}(\sigma(t)) \\
& =-k_{2} m(t) q(t)-\left(\sqrt{B(t)} w(\sigma(t))-\frac{A(t)}{2 \sqrt{B(t)}}\right)^{2}+\frac{A^{2}(t)}{4 B(t)} \\
& \leq-k_{2} m(t) q(t)+\frac{A^{2}(t)}{4 B(t)}, \quad t \geq t_{2} .
\end{aligned}
$$

Integrating (3.22) from $t_{2}$ to $t$, we get

$$
\int_{t_{2}}^{t}\left[k_{2} m(s) q(s)-\frac{A^{2}(s)}{4 B(s)}\right] \Delta s \leq w\left(t_{2}\right)-w(t) \leq w\left(t_{2}\right),
$$

which contradicts with (3.21).

Case (2). The proof of the case if (2.2) of Lemma 2.1 holds is similar to the proof of Theorem 3.1 and hence it is omitted. 
Theorem 3.3 Assume that the hypotheses of Theorem 3.1 hold, except (3.1). Moreover, suppose that, for every $t_{1}>t_{0}$,

(I) $\quad 0<\inf _{s \geq t_{1}}\left[\liminf _{t \rightarrow \infty} \frac{H(t, s)}{H\left(t, t_{1}\right)}\right]<\infty$,

(II) $\limsup _{t \rightarrow \infty} \frac{1}{H\left(t, t_{1}\right)} \int_{t_{1}}^{t} \frac{P^{2}(t, s)}{B(s)} \Delta s<\infty$,

and there exists $\psi \in C_{r d}(I)$ such that

(III) $\int_{t_{1}}^{\infty} \psi_{+}^{2}(\sigma(s)) B(s) \Delta s=\infty, \quad \psi_{+}(s)=\max \{\psi(s), 0\}$,

(IV) $\quad \limsup _{t \rightarrow \infty} \frac{1}{H\left(t, t_{1}\right)} \int_{t_{1}}^{t}\left[k_{2} m(s) q(s) H(t, s)-\frac{P^{2}(t, s)}{4 B(s)}\right] \Delta s \geq \psi\left(t_{1}\right)$.

Then every solution $y(t)$ of $(1.1)$ or $L_{2} y(t)$ is oscillatory.

Proof If $y$ is a nonoscillatory solution of $(1.1)$ on $\left[t_{1}, \infty\right)_{\mathbb{T}}$. Assume that $y(t)>0$ and $y(g(t))>$ 0 for $t \geq t_{1}$. By the proof of Lemma 2.1, we have that two cases of Lemma 2.1 hold.

Case (1). Suppose that (2.1) of Lemma 2.1 holds, then proceeding as in the proof of Theorem 3.1, we obtain (3.12), then

$$
\begin{aligned}
& \int_{t_{1}}^{t} k_{2} m(s) q(s) H(t, s) \Delta s \\
& \quad \leq H\left(t, t_{1}\right) w\left(t_{1}\right)+\int_{t_{1}}^{t} \frac{P^{2}(t, s)}{4 B(s)} \Delta s-\int_{t_{1}}^{t}\left[\sqrt{H(t, s)} \sqrt{B(s)} w(\sigma(s))+\frac{P(t, s)}{2 \sqrt{B(s)}}\right]^{2} \Delta s .
\end{aligned}
$$

By (IV), we get

$$
\begin{aligned}
\psi\left(t_{1}\right) \leq & \limsup _{t \rightarrow \infty} \frac{1}{H\left(t, t_{1}\right)} \int_{t_{1}}^{t}\left[k_{2} m(s) q(s) H(t, s)-\frac{P^{2}(t, s)}{4 B(s)}\right] \Delta s \\
\leq & w\left(t_{1}\right) \\
& -\liminf _{t \rightarrow \infty} \frac{1}{H\left(t, t_{1}\right)} \int_{t_{1}}^{t}\left[\sqrt{H(t, s)} \sqrt{B(s)} w(\sigma(s))+\frac{P(t, s)}{2 \sqrt{B(s)}}\right]^{2} \Delta s \quad \text { for all } t_{1} \geq t_{0},
\end{aligned}
$$

which implies that

$$
\psi(t) \leq w(t), \quad t \geq t_{0}
$$

and

$$
\liminf _{t \rightarrow \infty} \frac{1}{H\left(t, t_{1}\right)} \int_{t_{1}}^{t}\left[\sqrt{H(t, s)} \sqrt{B(s)} w(\sigma(s))+\frac{P(t, s)}{2 \sqrt{B(s)}}\right]^{2} \Delta s<\infty
$$

Now, define

$$
\begin{cases}c_{1}(t)=\frac{1}{H\left(t, t_{1}\right)} \int_{t_{1}}^{t} H(t, s) B(s) w^{2}(\sigma(s)) \Delta s, & t>t_{1}, \\ c_{2}(t)=\frac{1}{H\left(t, t_{1}\right)} \int_{t_{1}}^{t} \sqrt{H(t, s)} P(t, s) w(\sigma(s)) \Delta s, & t>t_{1} .\end{cases}
$$


It follows from (3.24) that

$$
\liminf _{t \rightarrow \infty}\left[c_{1}(t)+c_{2}(t)\right]<\infty
$$

Suppose that

$$
\int_{t_{1}}^{\infty} w^{2}(\sigma(s)) B(s) \Delta s=\infty
$$

i.e.,

$$
\lim _{t \rightarrow \infty} c_{1}(t)=\infty
$$

In fact, let $l$ be an arbitrary positive number. By condition (I) we can take a constant $\delta$ with

$$
\inf _{s \geq t_{1}}\left[\liminf _{t \rightarrow \infty} \frac{H(t, s)}{H\left(t, t_{1}\right)}\right]>\delta>0 .
$$

Since (3.26), there exists $T_{1}>t_{1}$ such that

$$
\int_{t_{1}}^{t} w^{2}(\sigma(\xi)) B(\xi) \Delta \xi \geq \frac{l}{\delta} \quad \text { for all } t \geq T_{1}
$$

Then, for every $t>t_{1}$, we have

$$
\begin{aligned}
c_{1}(t) & =\frac{1}{H\left(t, t_{1}\right)} \int_{t_{1}}^{t} H(t, s)\left[\int_{t_{1}}^{s} w^{2}(\sigma(\xi)) B(\xi) \Delta \xi\right]^{\Delta_{s}} \Delta s \\
& =\frac{1}{H\left(t, t_{1}\right)} \int_{t_{1}}^{t}\left[\int_{t_{1}}^{\sigma(s)} w^{2}(\sigma(\xi)) B(\sigma(\xi)) \Delta \xi\right]\left[-H^{\Delta_{s}}(t, s)\right] \Delta s,
\end{aligned}
$$

and consequently we have, for $t \geq T_{1}>t_{1}$,

$$
\begin{aligned}
c_{1}(t) & \geq \frac{1}{H\left(t, t_{1}\right)} \int_{T_{1}}^{t}\left[\int_{t_{1}}^{\sigma(s)} w^{2}(\sigma(\xi)) B(\sigma(\xi)) \Delta \xi\right]\left[-H^{\Delta_{s}}(t, s)\right] \Delta s \\
& \geq \frac{l / \delta}{H\left(t, t_{1}\right)} \int_{T_{1}}^{t}\left[-H^{\Delta_{s}}(t, s)\right] \Delta s=\frac{l}{\delta} \frac{H\left(t, T_{1}\right)}{H\left(t, t_{1}\right)} .
\end{aligned}
$$

But

$$
\liminf _{t \rightarrow \infty} \frac{H\left(t, T_{1}\right)}{H\left(t, t_{1}\right)}>\delta
$$

we can choose $T_{1}^{\prime} \geq T_{1}>t_{1}$ so that

$$
\frac{H\left(t, T_{1}\right)}{H\left(t, t_{1}\right)}>\delta
$$

for every $t \geq T_{1}^{\prime}$. Thus

$$
c_{1}(t) \geq l \quad \text { for all } t \geq T_{1}^{\prime},
$$

which proves (3.27), since $l>0$ is arbitrary. 
Next, we consider a sequence $\left(\varphi_{v}\right)_{v=1,2,3, \ldots}$ in the interval $\left(t_{1}, \infty\right)_{\mathbb{T}}$ with $\lim _{v \rightarrow \infty} \varphi_{v}=\infty$ and

$$
\lim _{v \rightarrow \infty}\left[c_{1}\left(\varphi_{v}\right)+c_{2}\left(\varphi_{v}\right)\right]=\liminf _{t \rightarrow \infty}\left[c_{1}(t)+c_{2}(t)\right]
$$

Since (3.25), there exists a constant $M$ so that

$$
c_{1}\left(\varphi_{v}\right)+c_{2}\left(\varphi_{v}\right) \leq M \quad(v=1,2,3, \ldots) .
$$

Furthermore, (3.27) guarantees that

$$
\lim _{v \rightarrow \infty} c_{1}\left(\varphi_{v}\right)=\infty
$$

Hence (3.29) implies

$$
\lim _{v \rightarrow \infty} c_{2}\left(\varphi_{v}\right)=-\infty
$$

From (3.29) and (3.30), for sufficiently large $v$, we derive

$$
1+\frac{c_{2}\left(\varphi_{v}\right)}{c_{1}\left(\varphi_{v}\right)} \leq \frac{M}{c_{1}\left(\varphi_{v}\right)}<\frac{1}{2} .
$$

Thus

$$
\frac{c_{2}\left(\varphi_{v}\right)}{c_{1}\left(\varphi_{v}\right)}<-\frac{1}{2} \quad \text { for all large } v,
$$

from (3.31),

$$
\lim _{v \rightarrow \infty} \frac{c_{2}^{2}\left(\varphi_{v}\right)}{c_{1}\left(\varphi_{v}\right)}=\infty
$$

On the other hand, by Hölder's inequality [18], for any positive integer $v$, we have

$$
\begin{aligned}
c_{2}^{2}\left(\varphi_{v}\right) & =\frac{1}{H^{2}\left(\varphi_{v}, t_{1}\right)}\left\{\int_{t_{1}}^{\varphi_{v}} P\left(\varphi_{v}, s\right) \sqrt{H\left(\varphi_{v}, s\right)} w(\sigma(s)) \Delta s\right\}^{2} \\
& \leq\left[\frac{1}{H\left(\varphi_{v}, t_{1}\right)} \int_{t_{1}}^{\varphi_{v}} P^{2}\left(\varphi_{v}, s\right) \frac{1}{B(s)} \Delta s\right]\left[\frac{1}{H\left(\varphi_{v}, t_{1}\right)} \int_{t_{1}}^{\varphi_{v}} H\left(\varphi_{v}, s\right) w^{2}(\sigma(s)) B(s) \Delta s\right] \\
& \leq\left[\frac{1}{H\left(\varphi_{v}, t_{1}\right)} \int_{t_{1}}^{\varphi_{v}} P^{2}\left(\varphi_{v}, s\right) \frac{1}{B(s)} \Delta s\right] c_{1}\left(\varphi_{v}\right),
\end{aligned}
$$

then

$$
\frac{c_{2}^{2}\left(\varphi_{v}\right)}{c_{1}\left(\varphi_{v}\right)} \leq \frac{1}{H\left(\varphi_{v}, t_{1}\right)} \int_{t_{1}}^{\varphi_{v}} P^{2}\left(\varphi_{v}, s\right) \frac{1}{B(s)} \Delta s \quad \text { for all large } v .
$$

By (3.28), we obtain

$$
\frac{H\left(\varphi_{v}, T_{1}\right)}{H\left(\varphi_{v}, t_{1}\right)}>\delta \quad \text { for sufficiently large } v,
$$


therefore

$$
\frac{c_{2}^{2}\left(\varphi_{v}\right)}{c_{1}\left(\varphi_{v}\right)} \leq \frac{1}{\delta} \frac{1}{H\left(\varphi_{v}, t_{1}\right)} \int_{t_{1}}^{\varphi_{v}} P^{2}\left(\varphi_{v}, s\right) \frac{1}{B(s)} \Delta s \quad \text { for all large } v .
$$

Because of (3.32), we get

$$
\lim _{v \rightarrow \infty} \frac{1}{H\left(\varphi_{v}, t_{1}\right)} \int_{t_{1}}^{\varphi_{v}} P^{2}\left(\varphi_{v}, s\right) \frac{1}{B(s)} \Delta s=\infty
$$

Thus

$$
\limsup _{t \rightarrow \infty} \frac{1}{H\left(t, t_{1}\right)} \int_{t_{1}}^{t} P^{2}(t, s) \frac{1}{B(s)} \Delta s=\infty,
$$

which contradicts with condition (II). We have thus proved that (3.26) fails. So, it holds that

$$
\int_{T_{0}}^{\infty} w^{2}(\sigma(s)) B(s) \Delta s<\infty
$$

By $(3.23)$ and $\psi_{+}(s)=\max \{\psi(s), 0\}$, we get

$$
\int_{T_{0}}^{\infty} \psi_{+}^{2}(\sigma(s)) B(s) \Delta s \leq \int_{T_{0}}^{\infty} w^{2}(\sigma(s)) B(s) \Delta s<\infty
$$

which yields a contradiction to condition (III). This completes the proof.

Case (2). The proof of the case if (2.2) of Lemma 2.1 holds is similar to the proof of Theorem 3.1 and hence it is omitted.

\section{Examples}

Example 4.1 As an illustrative example, we consider the following equation:

$$
y^{\prime \prime \prime}(t)+\frac{7}{4 t^{2}} y^{\prime}(2 t)+t^{-3} y\left(\frac{t}{2}\right)=0, \quad t \geq 2 .
$$

Here $\mathbb{T}=\mathbb{R}^{+}$, and $\alpha=\beta=1, r_{1}(t)=1, r_{2}(t)=1, p(t)=\frac{7}{4 t^{2}}, \psi(t, x)=x, a(t)=2 t, q(t)=t^{-3}$, $f(t, x)=x, g(t)=\frac{t}{2}, t_{0}=2$. By taking $m(t)=1, c=k_{1}=k_{2}=1, t_{1}=3, H(t, s)=(t-s)^{4}$, we have $Q(t)=-\frac{1}{4 t^{2}}$. And $R_{1}(t, 2)=R_{2}(t, 2)=\int_{2}^{t} 1 d s \rightarrow \infty$ as $t \rightarrow \infty$, we see that (1.2) and (1.3) are clearly satisfied. By Corollary 1 of [3], we obtain that the equation

$$
z^{\prime \prime}(t)+\frac{1}{4 t^{2}} z(2 t)=0
$$

is oscillatory. It is easy to check that all hypotheses of Theorem 3.1 are satisfied, so we get that equation (4.1) is oscillatory.

\section{Summary}

We present some new theorems for the oscillation of (1.1) by using the Riccati transformation, the integral averaging technique, and a new comparison theorem. Our method 
essentially simplifies the examination of the third order equation and, what is more, our results here extend and complement some of results of Bohner et al. In addition, the next step that can be done is as follows:

1. It would be of interest to consider (1.1) and try to obtain some oscillation criteria if $p(t)<0$ or $q(t)<0$.

2. We can consider the dynamic equation with advanced nonlinear term, that is, when $g(t)>t$ is considered.

\section{Acknowledgements}

The authors sincerely thank the reviewers for their valuable suggestions and useful comments that have led to the present improved version of the original manuscript.

Funding

This research is supported by the Natural Science Foundation of China (61703180), and supported by Shandong Provincial Natural Science Foundation (ZR2016AM17).

\section{Competing interests}

The authors declare that they have no competing interests

\section{Authors' contributions}

The authors declare that the study was realized in collaboration with the same responsibility. All authors read and approved the final manuscript.

\section{Publisher's Note}

Springer Nature remains neutral with regard to jurisdictional claims in published maps and institutional affiliations.

Received: 27 April 2018 Accepted: 28 May 2018 Published online: 05 July 2018

\section{References}

1. Liu, H., Meng, F.: Interval oscillation criteria for second-order nonlinear forced differential equations involving variable exponent. Adv. Differ. Equ. 2016, Article ID 291 (2016)

2. Shao, J., Zheng, Z., Meng, F.: Oscillation criteria for fractional differential equations with mixed nonlinearities. Adv. Differ. Equ. 2013, Article ID 323 (2013)

3. Baculíková, B.: Oscillatory behavior of the second order functional differential equations. Appl. Math. Lett. 72, 35-41 (2017)

4. Bohner, M., Grace, R., Sager, H., Tunc, E.: Oscillation of third-order nonlinear damped delay differential equations. Appl. Math. Comput. 278, 21-32 (2016)

5. Grace, S.R., Graef, J.R., El-Beltagy, M.A.: On the oscillation of third order neutral delay dynamic equations on time scales. Comput. Math. Appl. 63, 775-782 (2012)

6. Han, Z., Sun, S., Shi, B.: Oscillation criteria for a class of second-order Emden-Fowler delay dynamic equations on time scales. J. Math. Anal. Appl. 334, 847-858 (2007)

7. Li, T., Han, Z., Sun, S., Yang, D.: Existence of nonoscillatory solutions to second-order neutral delay dynamic equations on time scales. Adv. Differ. Equ. 2009, Article ID 562329 (2009)

8. Adivar, M., Akin, E., Higgins, R.: Oscillatory behavior of solutions of third-order delay and advanced dynamic equations. J. Inequal. Appl. 2014, Article ID 95 (2014)

9. Jadlovská, I.: Iterative oscillation results for second-order differential equations with advanced argument. Electron. J. Differ. Equ. 2017, Article ID 162 (2017)

10. Chen, D., Qu, P.L.: Oscillation of even order advanced type dynamic equations with mixed nonlinearities on time scales. J. Appl. Math. Comput. 44, 357-377 (2014)

11. Chen, D.: Oscillation of second-order Emden-Fowler neutral delay dynamic equations on time scales. Math. Comput. Model. 51, 1221-1229 (2010)

12. Erbe, L., Hassan, T., Peterson, A.: Oscillation criteria for nonlinear damped dynamic equations on time scales. Appl. Math. Comput. 203, 343-357 (2008)

13. Li, T., Han, Z., Sun, S., Zhao, Y.: Oscillation results for third order nonlinear delay dynamic equations on time scales. Bull. Malays. Math. Soc. 34, 639-648 (2011)

14. Bohner, M., Peterson, A.: Advances in Dynamic Equations on Time Scales. Birkhäuser, Boston (2003)

15. Philos, C.G.: Oscillation theorems for linear differential equations of second order. Arch. Math. 53, 482-492 (1989)

16. $\mathrm{Wu}, \mathrm{H}$., Erbe, L., Peterson, A.: Oscillation of solution to second-order half-linear delay dynamic equations on time scales. Electron. J. Differ. Equ. 2016, Article ID 71 (2016)

17. Gilbert, H.: Existence theorems for first-order equation on time scales with Caratheodory functions. Adv. Differ. Equ. 2010, Article ID 650827 (2010)

18. Chen, G.S., Chen, Z.: A functional generalization of the reverse Hölder integral inequality on time scales. Math. Comput. Model. 54, 2939-2942 (2011) 